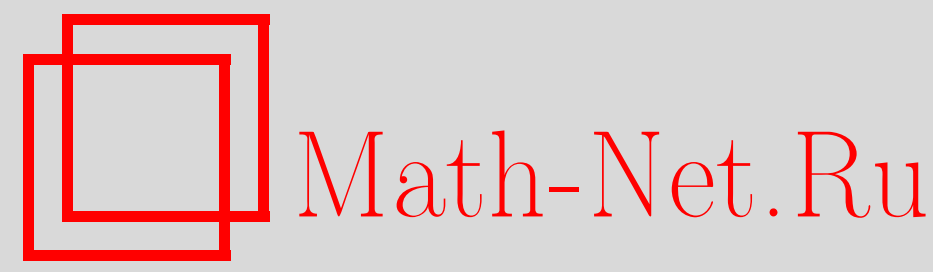

В. П. Варин, Отображение последования некоторых полиномиальных систем дифференциальных уравнений, Матем. сб., 2004, том 195, номер 7, 3-20

DOI: https://doi.org/10.4213/sm832

Использование Общероссийского математического портала Math-Net.Ru подразумевает, что вы прочитали и согласны с пользовательским соглашением http://www.mathnet.ru/rus/agreement

Параметры загрузки:

IP : 3.82 .47 .9

26 апреля 2023 г., 08:59:31 
УДК 517.5

\author{
В. П. Варин
}

\title{
Отображение последования некоторых полиномиальных систем дифференциальных уравнений
}

\begin{abstract}
В статье рассматривается один из подходов к классической проблеме различения центра и фокуса для систем дифференциальных уравнений на плоскости с полиномиальными правьми частями. Для шшрокого класса таких систем необходимые и достаточные условия центра выражаются с помощью уравнений в вариациях высокого порядка. В отличие от существующих методов исследования в этой работе основное внимание уделяется не получению условий центра как таковых, а вычислению асимптотики отображения последования (отображения Пуанкаре) в явном виде, что также дает возможность исследовать бифуркации рождения сколь угодно вырожденных циклов.

Библиограбиия: 14 названий.
\end{abstract}

K настоящему времени проблема различения центра и фокуса решена для некоторых частных случаев полиномиальных систем, среди которых можно выделить: системы с невырожденной линейной частью; системы, линейная часть которых является жордановой клеткой; системы, укорочение которых однородно и невырождено. Все эти случаи можно классифицировать как системы, у которых ломаная Ньютона состоит из одного ребра [1].

Сушествующие методы исследования этих систем сводятся либо к построению нормальной формы, либо к отысканию формального первого интеграла. Фокусные величины, которые при этом получаются, могут терять часть информации об асимптотике отображения последования исходной системы. Метод вычисления фокусных величин, предложенньй в этой статье, полностью сохраняет эту информацию и позволяет вычислить необходимое их количество в аналитическом виде или численно.

Существует также подход к проблеме центр-фүокус, основанный на вычислении обобщенной фокусной величины [2]. При этом главный член асимптотики преобразования монодромии всегда линеен и зависит от трансверсали. Разумеется, информация о реальных фокусных величинах при этом также теряется.

В п. 1 рассматривается более широкий класс полиномиальных систем ОДУ (у которых ломаная Ньютона состоит из одного ребра), чем системы, указанные выше. Вводится обобшенная полярная замена координат, пригодная для всего класса таких систем, и даются условия, при которых особая точка в нуле является монодромной. В отличие от полярного раздутия (см. [3]) при такой замене не возникают новые особые точки на вклеенной окружности.

Работа вьполнена при поддержке Российского фонда фундаментальных исследований (гранты № № 02-01-01067, 01-01-00517).

(C) В.П. ВАрин 2004 
В п. 2 приводится процедура вычисления уравнений в вариациях высокого порядка для ОДУ, полученных в результате обобщенной полярной замены координат. Показано, что все уравнения в вариациях на нулевом решении интегрируются в квадратурах и, таким образом, асимптотика отображения последования вычисляется в явном виде. Доказана теорема о почти алгебраической разрешимости проблемы центр-фокус для любых систем класса, введенного в п. 1.

В п. 3 рассматриваются системы ОДУ, близкие к гамильтоновьм системам на плоскости, у которых ломаная Ньютона состоит из одного ребра. Вводятся замены координат, при которых траектория укороченной, т.е. гамильтоновой, системы преобразуется в окружность, а возмушения отслеживаются как деформации этой окружности.

В п. 4 замены координат, предложенные в п. 3, обобщаются на некоторые системы ОДУ с ломаной Ньютона, состоящей из двух ребер. Впервые для таких систем получены условия центра вместе с асимптотикой отображения последования. Показано, что такие случаи сводятся к исследованию систем ОДУ на римановых поверхностях.

1. Обобщенная полярная замена координат. Рассмотрим системы ОДУ, ломаная Ньютона которых состоит из одного ребра и которые с помощью перенормировки $x, y$ и $t$ приводятся к виду

$$
\begin{aligned}
& \frac{d x}{d t} \stackrel{\text { def }}{=} \dot{x}=p(x, y) \stackrel{\text { def }}{=} y^{2 j m-1}+\cdots, \\
& \frac{d y}{d t} \stackrel{\text { def }}{=} \dot{y}=q(x, y) \stackrel{\text { def }}{=}-x^{2 j n-1}+\cdots,
\end{aligned}
$$

где $j, m, n \in \mathbb{N}, m$ и $n$ взаимно просты и многоточие обозначает мономы, все векторные показатели которых лежат правее ребра ломаной Ньютона [1], [4] системы (1.1) (следуя Ляпунову [5] это случай первой категории) либо правее и на ребре ломаной Ньютона системы (1.1) (случай второй категории). Ляпунов рассмотрел системы (1.1) для $j=m=1$ [5].

ЗАмЕчАнИЕ 1.1. Укорочение системы (1.1) первой категории - это гамильтонова система, имеющая центр в нуле. Интегральные кривые этой системы

$$
\frac{x^{2 j n}}{n}+\frac{y^{2 j m}}{m}=\mathrm{const}
$$

вытянуты вдоль одной из осей координат (если $m \neq n$ ) таким образом, что при стягивании фазовой кривой к началу координат отношение минимального и максимального диаметров фигуры, образованной фазовой кривой, стремится к нулю. Ясно, что обычная полярная замена координат в этом случае сингулярна.

Для системы (1.1) сделаем обобщенную полярную замену координат:

$$
x=r^{m} \cos \varphi, \quad y=r^{n} \sin \varphi
$$

где $m$ и $n-$ те же, что и в системе (1.1). Тогда система (1.1) принимает вид

$$
\frac{d r}{d \varphi} \stackrel{\text { def }}{=} r^{\prime}=\frac{r\left(r^{n} p(x, y) \cos \varphi+r^{m} q(x, y) \sin \varphi\right)}{m r^{m} q(x, y) \cos \varphi-n r^{n} p(x, y) \sin \varphi} .
$$


Замена переменных, предложенная Ляпуновым для систем (1.1) с $j=m=1$, использует введенные им специальные функции. Это позволяет упростить некоторые формулы, но требует исследования интегралов от этих специальных функций. Ллпунов сводит эти интегралы к нескольким типам, которые требуют отдельного исследования [5; п. 13].

Согласно [1], [6] назовем степенъю монома $x^{k} y^{l}, k, l \in \mathbb{N}$, в первом уравнении системы (1.1) его квазиоднородную степень $(k-1) m+l n$, а во втором уравнении системы $(1.1)$ - его квазиоднородную степень $k m+(l-1) n$. Очевидно, в случае первой категории степень мономов системы (1.1), отмеченных многоточием, превосходит $2 j m n-n-m$. Если же некоторые мономы системы (1.1) имеют степень $2 j m n-n-m$, то эти показатели степени лежат на ребре ломаной Ньютона системы (1.1) и это случай второй категории.

Определим функцию

$$
F(\varphi)=\lim _{r \rightarrow 0} \frac{r^{\prime}}{r}
$$

где $r^{\prime}$ - это правая часть уравнения (1.3). Очевидно, этот предел существует.

ТЕОРЕМа 1.1. Если знаменатель функиии $F(\varphi)$ не обращается в нуль для достаточно малых $|\operatorname{Im} \varphi|$, то особая точка нуль системы (1.1) монодромна.

ДокАЗАТЕЛЬСтво. Числитель и знаменатель функции $F(\varphi)$ суть тригонометрические полиномы с коэффициентами мономов степени $2 j m n-n-m$ системы (1.1), причем знаменатель функции $F(\varphi)$, очевидно, удовлетворяет условию теоремы при достаточно малых коэффициентах мономов укороченной системы (1.1). Разложим правую часть уравнения (1.3) в ряд Тейлора по $r$. Каждый член этого разложения является дробью, знаменатель которой - некоторая степень знаменателя функции $F(\varphi)$. Следовательно, правая часть уравнения (1.3) аналитична для достаточно малых $|r|$ и $|\operatorname{Im} \varphi|$, поэтому решение ОДУ (1.3) сушествует для $\varphi \in[0,2 \pi]$, т.е. особая точка монодромна.

Например, для системы $(j=1, m=1, n=2) \dot{x}=y+a x^{2}, \dot{y}=-x^{3}$

$$
F(\varphi)=-\frac{\cos \varphi\left(a \cos ^{2} \varphi+\sin ^{3} \varphi\right)}{\cos ^{4} \varphi+2 a \cos ^{2} \varphi \sin \varphi+2 \sin ^{2} \varphi}
$$

Знаменатель $\cos ^{4} \varphi+2 a \cos ^{2} \varphi \sin \varphi+2 \sin ^{2} \varphi \neq 0$ при условии $|a|<\sqrt{2}$.

Заметим, что если знаменатель функции $F(\varphi)$ имеет кратные корни, то особая точка нуль системы (1.1) второй категории может быть монодромной [1], [7]. Это может быть, например, в случае, когда система (1.1) получена поворотом осей координат из некоторой системы, у которой ломаная Ньютона состоит из двух или более ребер.

2. Уравнения в вариациях. Алгоритм вычисления уравнений в вариациях произвольного порядка для уравнения $r^{\prime}=f(r, \varphi)$ состоит в следующем [6], [8]. Запишем это уравнение как уравнение в вариациях нулевого порядка, сделав в нем подстановку $r=r_{0}$, т.е. $r_{0}^{\prime}=f\left(r_{0}, \varphi\right)$. Пусть $r_{0}(\varphi)$ является решением этого 
уравнения с начальным значением $\rho=r_{0}(0) \neq 0$. Введем обозначение $r_{k}(\varphi)=$ $\partial r_{k-1}(\varphi) / \partial \rho, k=1,2, \ldots$ Формальным дифференцированием находим уравнения в вариациях:

$$
r_{k}^{\prime}=\frac{\partial f_{k-1}\left(r_{0}, \ldots, r_{k-1}, \varphi\right)}{\partial \rho}=f_{k}\left(r_{0}, \ldots, r_{k}, \varphi\right)=\frac{\partial^{k} f\left(r_{0}, \varphi\right)}{\partial \rho^{k}}
$$

Начальные значения для решений уравнений в вариациях $r_{k}(\varphi)$ суть $r_{1}(0)=1$ и $r_{k}(0)=0, k=2,3, \ldots$

ТЕОРема 2.1. Пусть выполнено условие теоремъ 1.1. Особая точка нуль системы (1.1) является центром тогда и только тогда, когда все уравнения в вариациях на нулевом решении уравнения (1.3) имеют в качестве решений $2 \pi$-периодические функции, не имеющие полюсов на вещественной оси.

ДокАЗАтЕльство. На плоскости $\mathbb{R}^{2}$ рассмотрим характеристическое множество уравнения (1.3) [6], [8]: $\chi=\{r(0), r(2 \pi)\}$ для достаточно малых начальных значений $r(0)$. Согласно теореме 1.1 множество $\chi$ является аналитической кривой, проходящей через начало координат (см. рис. 1). Очевидно, особая точка нуль системы (1.1) является центром тогда и только тогда, когда множество $\chi$ является биссектрисой первого квадранта плоскости $\mathbb{R}^{2}$.

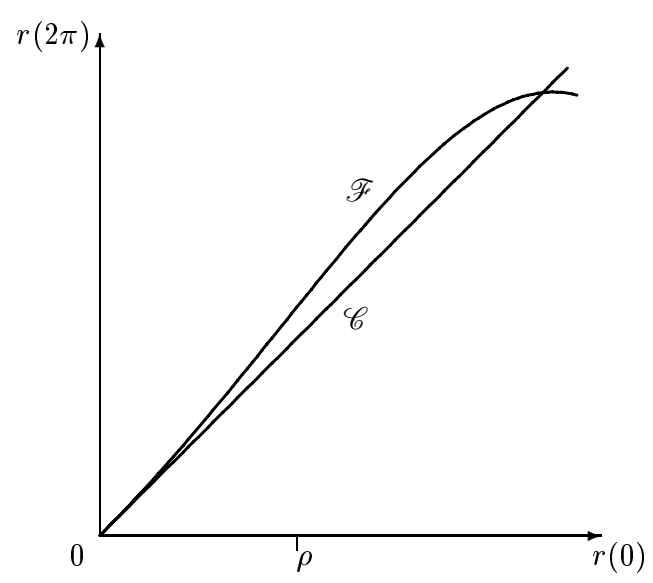

Рис. 1. Характеристическое множество центра $(\mathscr{C})$ и фокуса $(\mathscr{F})$

Пусть $r_{k}(\varphi), k=1,2, \ldots,-$ решения уравнений в вариациях и $\rho=r_{0}(0) \neq 0$ это начальное значение решения уравнения (1.3). Величины $r_{k}(2 \pi), k=1,2, \ldots$, являются значениями производных к кривой $\chi$ в точке $\rho[8]$ (см. рис. 1$)$. Все решения уравнений в вариациях зависят аналитически от начального значения $\rho$ согласно теореме 1.1. Поэтому величины $r_{k}(2 \pi), k=1,2, \ldots$, для уравнений в вариациях на нулевом решении уравнения (1.3) являются значениями производных к кривой $\chi$ в нуле.

Аналитическая кривая является биссектрисой первого квадранта плоскости $\mathbb{R}^{2}$ тогда и только тогда, когда имеет в нуле те же производные, т.е. $r_{1}(2 \pi)=1$, $r_{k}(2 \pi)=0, k=2,3, \ldots$. Эти величины совпадают с начальными значениями 
решений уравнений в вариациях, поэтому достаточность условия теоремы доказана.

Далее мы будем иметь дело только с уравнениями в вариациях на нулевом решении.

Докажем необходимость. Первое уравнение в вариациях имеет вид

$$
r_{1}^{\prime}=r_{1} F(\varphi)
$$

где $F(\varphi)$, определенная формулой $(1.4)$, является $2 \pi$-периодической функцией. Поэтому если $r_{1}(2 \pi)=1$, то $r_{1}(\varphi)$ - также $2 \pi$-периодическая функция с теми же свойствами. Очевидно,

$$
r_{1}(\varphi)=\exp \left(\int_{0}^{\varphi} F(\phi) d \phi\right) .
$$

По индукции $k$-е уравнение в вариациях имеет вид

$$
r_{k}^{\prime}=r_{k} F(\varphi)+G_{k}(\varphi), \quad k=2,3, \ldots
$$

где $G_{k}(\varphi)$ - это $2 \pi$-периодическая функция, не имеющая полюсов на вещественной оси. Поэтому если $r_{k}(2 \pi)=0$, то $r_{k}(\varphi)$ - также $2 \pi$-периодическая функция с теми же свойствами. Очевидно,

$$
r_{k}(\varphi)=r_{1}(\varphi) \int_{0}^{\varphi} \frac{G_{k}(\phi)}{r_{1}(\phi)} d \phi .
$$

На самом деле, $G_{k}(\varphi)$ делится на $r_{1}^{2}(\varphi)$, что следует из формулы $(2.4)$. Попутно доказано, что все уравнения в вариациях интегрируются в квадратурах.

ЗАмечАниЕ 2.1. Расположение кривой $\chi$ по отношению к биссектрисе $\mathscr{C}$ позволяет судить об устойчивости или неустойчивости фокуса, а также о наличии предельных циклов и об их устойчивости в системе координат (1.2). Например, кривая $\mathscr{F}$ на рис. 1 соответствует неустойчивому фокусу, а точка пересечения $\mathscr{F} \cap \mathscr{C}$ соответствует устойчивому предельному циклу уравнения (1.3). Для исходной системы устойчивость и неустойчивость меняются местами, если обход особой точки нуль совершается по часовой стрелке.

ЗАмечАниЕ 2.2. Для бесконечно гладких систем не существует теоремы, аналогичной теореме 2.1. Для таких систем возможна ситуация, когда кривые $\chi$ и $\mathscr{C}$ имеют бесконечньй порядок касания в нуле, но не совпадают (например, для линейного осциллятора с экспоненциально малым трением [6]). Возможна также ситуация центрофокуса [7], когда кривая $\chi$ пересекает кривую $\mathscr{C}$ бесконечное число раз в любом интервале значений $0<r(0)<\varepsilon \rightarrow 0$. Каждое такое пересечение соответствует, как было отмечено, предельному циклу.

Таким образом, величины $r_{k}(2 \pi), k=1,2, \ldots$, являются фокусными для монодромной особой точки системы (1.1). Если $r_{1}(2 \pi) \neq 1$, то имеет место грубый фокус (первого порядка по определению), т.е. кривая $\chi$ трансверсальна биссектрисе $\mathscr{C}$ в начале координат. Для случая первой категории фокус, очевидно, не может быть грубым. Если $r_{1}(2 \pi)=1, r_{l}(2 \pi)=0, l<k$, но $r_{k}(2 \pi) \neq 0$, тогда имеет место негрубый фокус (порядка $k$ по определению). Очевидно, порядок негрубого фокуса соответствует порядку касания кривых $\chi$ и $\mathscr{C}$ в начале координат. 
ЗАмечание 2.3. Для системы (1.1) с однородным укорочением $(m=n)$ в случае негрубого фокуса процедура вычисления фокусных величин $r_{k}(2 \pi)$ равносильна процедуре вычисления коэффициентов нормальной формы в [1; гл. II, п. 3.2], если в доказательстве теоремы 2.1 ввести обобшенный полярный радиус $R_{0}=$ $r_{0} / r_{1}$, что означает приведение укороченной системы к нормальной форме [1]. При этом решения уравнений в вариациях для обобшенного радиуса суть $R_{k}=r_{k} / r_{1}$, $k=1,2, \ldots$.

Следуюшая теорема позволяет оценить порядок фокуса для монодромной особой точки системы (1.1) по степеням ее мономов, не вычисляя уравнения в вариациях.

ТЕОРема 2.2. Пусть выполнено условие теоремъ 1.1 и особая точка нуль системь (1.1) является фокусом порядка $N$. Если в системе (1.1) опустить мономы степени, превосходящей $2 j m n-n-m+N-1$, то полученная таким образом система также имеет фокус порядка $N$.

ДокАЗАТЕЛЬСТво. Самая низкая степень мономов в системе (1.1) равна $2 j m n-$ $n-m$. Все коэффициенты мономов, имеюших эту степень, входят в первое и последующие уравнения в вариациях. Коэффициенты мономов степени $2 j m n-n-m+1$ входят во второе и последующие уравнения в вариациях. И так далее до мономов степени $2 j m n-n-m+N-1$, коэффициенты которых входят в $N$-е уравнение в вариациях. Коэффициенты мономов большей степени не участвуют в вычислении величин $r_{k}(2 \pi), k \leqslant N$, что следует из доказательства теоремы 2.1.

Следуюшая теорема рассматривает фокусные величины как функции коэффициентов мономов системы (1.1). Равенство нулю фокусной величины (начиная со второй) - это условие чентра. Напомним, что почти алгебраическая разрешимость - это алгебраическая разрешимость при фиксированных коэффициентах мономов укороченной системы [1], [9].

ТЕОРема 2.3. Пусть для системы (1.1) выполнено условие теоремы 1.1. Тогда проблема иентр-фокус почти алгебраччески разрешима; для случая первой категории все фокуснье величинь алгебраичны; для случая второй категории условие негрубости фокуса алгебраично и все фокусные величинь, начиная со второй, трансцендентнь.

ДокаЗАтельство. Рассмотрим случай первой категории. Функция $r_{1}(\varphi)$ в уравнении (2.2) не зависит от коэффициентов системы (1.1). Знаменатели функций $G_{k}(\varphi)$ также не зависят от коэффициентов системы (1.1) (см. доказательство теоремы 1.1). Поэтому если считать предыдущие условия центра вьполненными, то под знаком интеграла в формуле (2.4) находятся периодические функции, умноженные на полиномы от коэффициентов мономов системы (1.1), т.е. $k$-я фокусная величина - это полином от коэффициентов мономов системы (1.1). Почти алгебраическая разрешимость доказьвается аналогично (теорема 2 в $[9 ; \S 3]$ является частным случаем).

Для полиномиальных систем (1.1) может быть лишь конечное число независимых условий центра в силу теоремы Гильберта о базисах в полиномиальных идеа$\operatorname{лаx~[10].~}$ 
Рассмотрим теперь случай второй категории. Условие негрубости фокуса - это равенство нулю интеграла по периоду $2 \pi$ от функции $F(\varphi)$. Числитель и знаменатель этой функции суть тригонометрические полиномы с коэффициентами мономов укороченной системы (1.1). Сделаем подстановку $u=\operatorname{tg}(\varphi / 2)$ и преобразуем этот интеграл к виду

$$
\int_{-\infty}^{\infty} R(u) \frac{d u}{1+u^{2}}
$$

где $R(u)$ - рациональная функция от $u$, не имеющая полюсов на интервале $(-\infty, \infty)$ в силу условия теоремы 1.1. Разложим $R(u)$ на простые дроби. При этом возникают алгебраические функции коэффициентов системы (1.1) (т.е. корни некоторого полинома от $и$ с коэффициентами из полиномов от коэффициентов системы (1.1)), поэтому в результате интегрирования получим алгебраическую функцию от коэффициентов системы (1.1).

Заметим, что сама фокусная величина $r_{1}(2 \pi)$ при этом может быть как алгебраической, так и трансцендентной функцией коэффициентов мономов укороченной системы.

Все последующие фокусные величины системы (1.1) второй категории зависят от условия монодромности особой точки нуль укороченной системы (1.1). Это условие, т.е. неравенство нулю знаменателя функции $F(\varphi)$, определяет границы допустимых значений коэффициентов мономов укороченной системы $(1.1)$, при которых ее особая точка нуль монодромна. Для каждого коэффициента границы его допустимых значений являются полюсами функции $F(\varphi)$, а для функции $r_{1}(\varphi)$ они являются существенно особыми точками, так как входят в экспоненту (2.2). Функции $G_{k}(\varphi)$ в формуле $(2.4)$ делятся на $r_{1}^{2}(\varphi)$, т.е. под знаком интеграла в формуле (2.4) стоят функции, имеющие существенно особые точки. Следовательно, если $k$-я фокусная величина зависит от некоторого коэффициента монома укороченной системы (1.1), то границы интервалов его допустимых значений (при фиксированных остальных коэффициентах) являются сушественно особьми точками функции $r_{k}(2 \pi)$, т.е. эта функция не может быть алгебраичной.

Теорема 2.3 обобщает результат Ляпунова для систем (1.1) с $j=m=1[5$; пп. 9-18]. Заметим, что все условия центра для систем (1.1) с $j=m=1$ алгебраичны [5], [11].

Алгебраичность фокусной величины, очевидно, влечет алгебраичность условия центра, но условия центра могут быть алгебраичными и для трансцендентных фокусных величин (см. пример 2.2).

ПримеР 2.1. Рассмотрим систему уравнений с квадратичной нелинейной частью. Вращением осей координат приведем ее к виду [12]

$$
\begin{aligned}
& \dot{x}=-y-b x^{2}-C x y-d y^{2}, \\
& \dot{y}=x+a x^{2}+A x y-a y^{2} .
\end{aligned}
$$

В работе [12] были получены необходимые условия центра системы (2.5) в виде базиса Грёбнера для идеала кольца полиномов от коэффициентов этой системы, порожденного константами Пуанкаре-Ляпунова. Достаточность этих условий доказывалась независимо. 
Константы Пуанкаре-Ляпунова вычислялись в процессе вычисления формального (и аналитического) первого интеграла системы (2.5), причем в их определении существовал некоторый произвол [12], т.е. асимптотика отображения последования при этом не вычислялась.

Заметим, что если линейная часть ОДУ отсутствует или вырождена, то формальный первый интеграл может не существовать (см. пример 2.3), в то время как теорема 2.1 не имеет этих ограничений.

Вычисления с помощью уравнений в вариациях оказываются более простьми, хотя также требуют привлечения методов компютерной алгебры. Необходимо вычислить семь уравнений в вариациях [6]. Первое уравнение тривиально, т.е. $r_{1}(\varphi) \equiv 1$. Решения всех последующих уравнений в вариациях суть тригонометрические полиномы (т.е. отрезки ряда Фурье), так как непериодическую часть функций $r_{k}(\varphi), k=2, \ldots$, следует отбрасывать на каждом шаге по теореме 2.1 .

Вычисляя базис Грёбнера для фокусных величин, получаем три необходимых (и достаточных [12]) условия центра системы (2.5):

$$
\begin{aligned}
& 0=(2 a+C)(b+d), \\
& 0=(A+3 b+5 d)(2 b-A)(b+d) C, \\
& 0=\left(4(d-A) d+3 C^{2}\right)(2 b-A)(b+d)(2 d+A) C .
\end{aligned}
$$

Созданное программное обеспечение в системе компьютерной алгебры Maple позволило провести все вычисления, включая базис Грёбнера (2.6), менее чем за 5 секунд на компютере Pentium III, 500 MHz.

Заметим, что для системы с общей кубической нелинейной частью (т.е. включающей квадратичные и кубичные члены) объем вычислений столь велик, что не удается вычислить даже необходимое количество фокусных величин, не говоря уже о базисе Грёбнера (см. обзор результатов в этом направлении в [13]).

ПримеР 2.2. Рассмотрим систему уравнений

$$
\dot{x}=y+a x^{2}, \quad \dot{y}=-x^{3}+b x^{2} y
$$

Система (2.7) инвариантна при замене $(x, y, a) \rightarrow(-x,-y,-a)$, поэтому достаточно рассматривать $a \geqslant 0$. Сделаем замену переменных (1.2) с $m=1, n=2$ и вычислим четыре уравнения в вариациях. Моном $b x^{2} y$ имеет вторую степень и не влияет на функцию $F(\varphi)$, поэтому $F(\varphi)$ для системы $(2.7)$ - это функция (1.5).

При $b=0$ система (2.7) обратима, т.е. инвариантна при замене переменных $(x, y, t) \rightarrow(-x, y,-t)$, поэтому по теореме 2.2 фокус в системе (2.7) не может быть грубым.

Функция $G_{2}(\varphi)($ см. формулу $(2.4))$ имеет вид

$$
G_{2}(\varphi)=-\frac{2 b r_{1}^{2}(\varphi) \cos ^{2} \varphi \sin \varphi\left(1+\sin ^{2} \varphi\right)\left(a \cos ^{2} \varphi+\sin \varphi\right)}{\left(\cos ^{4} \varphi+2 a \cos ^{2} \varphi \sin \varphi+2 \sin ^{2} \varphi\right)^{2}}
$$

где $r_{1}(\varphi)$ находится по формулам (1.5), (2.2). Поэтому вторая фокусная величина имеет вид

$$
r_{2}(2 \pi)=r_{2}(a, b)=\int_{0}^{2 \pi} \frac{G_{2}(\varphi)}{r_{1}(\varphi)} d \varphi
$$


При $a=0$ фокусная величина $r_{2}(0, b)$ выражается через эллиптические интегралы (см. пример 3.2). Далее положим $b=1$, так как $r_{2}(a, b)$ зависит от $b$ линейно.

Функция $r_{2}(a)$ трансцендентна, так как имеет существенно особые точки при $a= \pm \sqrt{2}$ (см. также [5; п. 20]).

Расчеты показали, что система (2.7) имеет в нуле фокус второго порядка при всех $a<\sqrt{2}$, кроме $a=a_{0} \approx 0.912871$. При этом значении параметра величина $r_{2}(a)$ меняет знак, т.е. $r_{2}(a)<0$ при $a<a_{0}$ и $r_{2}(a)>0$ при $a_{0}<a<\sqrt{2}$. Кроме того, $r_{2}(a) \asymp\left(a-a_{0}\right) C_{2}, r_{3}(a) \asymp\left(a-a_{0}\right)^{2} C_{3}$ и $r_{4}(a) \asymp C_{4}$ при $a \rightarrow a_{0}$, где $r_{3}(a)$ и $r_{4}(a)$ - соответственно третья и четвертая фокусные величины, $C_{2}, C_{3}$, $C_{4}$ - положительные константы, которые могут быть вычислены с той же точностью, что и величины $r_{k}(a)$, если рассматривать также смешанные вариации уравнений $(2.1),(2.3)$ по коэффициенту $a[7]$.

Следовательно, в системе координат (1.2) фокус неустойчив при $a>a_{0}$, так как в окрестности нуля $r(2 \pi)>r(0)$. Соответственно при $a<a_{0}$ фокус устойчив, так как $r(2 \pi)<r(0)$ при малых $r(0)$. Поэтому при $a<a_{0}$ система $(2.7)$ имеет неустойчивый предельный цикл в системе координат (1.2) (см. рис. 1). В исходных координатах устойчивость и неустойчивость меняются местами, так как обход вокруг нуля происходит по часовой стрелке.

Оценим началъный радиус предельного цикла, т.е. величину $r(2 \pi)=r(0)=$ $r(0)(a)$ при малых $a-a_{0}<0$. Разложение величины $r(2 \pi)$ для уравнения (1.3) по начальному значению $\rho=r(0)$ в окрестности нуля (см. рис. 1$)$ имеет вид

$$
r(2 \pi)=\rho+\frac{r_{2}(a) \rho^{2}}{2 !}+\frac{r_{3}(a) \rho^{3}}{3 !}+\frac{r_{4}(a) \rho^{4}}{4 !}+\cdots,
$$

поэтому

$$
r(0)(a) \approx 2 \frac{-r_{3}(a)+\sqrt{r_{3}^{2}(a)-3 r_{2}(a) r_{4}(a)}}{r_{4}(a)}
$$

т.e. $r(0)(a) \asymp \sqrt{a_{0}-a}$.

Например, $r(0)\left(a_{1}\right) \approx 0.15278007$ при $a_{1}=a_{0}-0.05$ (устойчивый предельный цикл системы $(2.7))$. При этом $r_{2}\left(a_{1}\right) \approx-0.05147115, r_{3}\left(a_{1}\right) \approx 0.00397391$, $r_{4}\left(a_{1}\right) \approx 25.168555$. По формуле $(2.9)$ получаем $r(0)\left(a_{1}\right) \approx 0.15633923$.

На самом деле, корень уравнения $r_{2}(a)=0$ - это $a_{0}=\sqrt{5 / 6}$, что можно получить методом Ляпунова [5; пा. 19, 20] или преобразуя систему $(2.7)$ с помощью формальных рядов [11], однако информация о фокусных величинах при этом теряется.

ПримеР 2.3. Рассмотрим систему уравнений

$$
\dot{x}=y^{3}+a x^{4}+b x^{2} y^{2}, \quad \dot{y}=-x^{3}+c x^{2} y^{2} .
$$

Эта система при $a=0, b=0$ и $c=1 / 2$ приведена в [12; c. 462] в качестве примера системы, не имеющей ни аналитического, ни формального первого интеграла, т.е. метод вычисления констант Пуанкаре-Ляпунова к этой системе не применим.

Сделаем обычную полярную замену координат. Система (2.10) первой категории, поэтому фокус не может быть грубым. Из доказательства теоремы 2.1 следует, что $k$-я фокусная величина системы (2.10) - это однородный полином от величин 
$a, b, c$ степени $k-1$, коэффициенты которого вычисляются явно в виде интегралов от периодических функций по периоду. В частности, вторая фокусная величина это линейная функция от $a, b, c$, и поэтому она тождественно равна нулю, так как система (2.10) обратима, если любые два коэффициента из $a, b, c$ равны нулю.

Заметим, что нет необходимости вычислять интегралы по квадратурным формулам, что крайне неэффективно в вычислительном отношении. Вместо этого следует интегрировать совместно систему уравнений в вариациях $(2.1),(2.3)$ до нужного порядка.

Приведем приближенные значения фокусных величин системы (2.10) до пятого порядка:

$$
\begin{aligned}
r_{3} \approx & -2.03331140 a c, \quad r_{4} \approx 1.94895376 a c^{2}, \\
r_{5} \approx & c\left(20.671776 a^{2} c-170.89237 a^{2} b-160.86409 a^{3}+21.411152 a c^{2}\right. \\
& \left.\quad-13.731442 b^{3}+13.731442 b c^{2}-77.112488 a b^{2}\right) .
\end{aligned}
$$

ПримеР 2.4. Рассмотрим систему уравнений

$$
\dot{x}=y^{3}+a x^{3} y+b x^{5}, \quad \dot{y}=-x^{5}+c x^{2} y^{2} .
$$

Системы этого типа в контексте проблемы центр-фокус ранее нигде не рассматривались. Сделаем замену переменных (1.2) с $m=2, n=3$. Функция $F(\varphi)$ для системы (2.11) имеет вид

$$
F(\varphi)=-\frac{\sin \varphi \cos \varphi\left(\sin ^{2} \varphi+a \cos ^{3} \varphi+c \sin ^{2} \varphi \cos \varphi-\cos ^{4} \varphi\right)}{2 \cos ^{6} \varphi+(3 a-2 c) \sin ^{2} \varphi \cos ^{3} \varphi+3 \sin ^{4} \varphi} .
$$

По теореме 1.1 особая точка нуль системы (2.11) монодромна при условии $|3 a-2 c|<2 \sqrt{6}$. Укороченная система (при $b=0$ ) обратима $(x, y, t \rightarrow x,-y,-t)$, и ее мономы имеют степень 7. Моном $b x^{5}$ имеет степень 8 . Следовательно, фокус в системе (2.11) не может быть грубым.

Положим $c=3 a / 2$ и исследуем рождение предельных циклов в системе $(2.11)$ при изменении параметра $a$.

Функция $G_{2}(\varphi)$ (см. формулу (2.4)) имеет вид

$$
G_{2}(\varphi)=\frac{b r_{1}^{2}(\varphi) \cos ^{7} \varphi\left(2+\sin ^{2} \varphi\right)\left(3 a \sin ^{2} \varphi-2 \cos ^{3} \varphi\right)}{\left(2 \cos ^{6} \varphi+3 \sin ^{4} \varphi\right)^{2}}
$$

где $r_{1}(\varphi)$ находится по формуле (2.2). Вторая фокусная величина $r_{2}(a, b)$ вычисляется по формуле (2.8). Она зависит от $b$ линейно, поэтому далее положим $b=1$.

Исследование предельных циклов системы (2.11) в точности повторяет это исследование для системы (2.7), начиная с формулы (2.8). Отличие состоит в том, что при $c=3 a / 2$ особая точка нуль системы (2.11) монодромна при любом $a$, а также в численных значениях констант. Приведем эти значения.

Критическое значение параметра $a$, при котором величина $r_{2}(a)$ меняет знак, $a_{0} \approx 4.54132378$ (см. пример 3.3). При $a-a_{0}<0$ система (2.11) имеет устойчивый предельный цикл. Возьмем $a=a_{1}=a_{0}-0.1$. Начальный радиус предельного цикла $r(0)\left(a_{1}\right) \approx 0.57026001$. При этом $r_{2}\left(a_{1}\right) \approx-0.03689080, r_{3}\left(a_{1}\right) \approx 0.00204139$, $r_{4}\left(a_{1}\right) \approx 1.22542310$. По формуле $(2.9) r(0)\left(a_{1}\right) \approx 0.59772201$.

Другой пример вырожденной бифуркации цикла, в котором требуется выгислять решения шести уравнений в вариациях, см. в [6]. 
3. Системы, близкие к гамильтоновым. Рассмотрим системы (1.1) первой категории как негамильтоновы возмущения укороченной гамильтоновой системы [14]. Нас интересует сколь угодно малая окрестность нуля системы (1.1), поэтому величина коэффициентов системы (1.1) не имеет значения.

Обобщенная полярная замена координат (1.2), очевидно, не является единственно возможной для систем (1.1). Ее смысл состоит в том, что новые особенности не возникают, как это имеет место при полярном раздутии. Замену координат (1.2) можно интерпретировать как отображение плоскости $\mathbb{R}^{2}$ в себя, при котором индуцированная система допускает обычную полярную замену координат (см. [1; 1 , пп. 1.1, 1.2]). Таким образом, можно строить замены координат, при которых траектория невозмушенной гамильтоновой системы является наиболее простой, т.е. окружностью.

Замена координат, предложенная Ляпуновым для систем (1.1) первой категории с $j=m=1$ [5], обладает этим свойством. Однако специальные функции Ляпунова, вообше говоря, не интегрируются в явном виде, поэтому практическое использование этой замены затруднительно.

С точностью до перенормировки $x, y$ и $t$ гамильтониан укороченной системы (1.1) имеет вид

$$
H=\frac{x^{2 j n}}{j n}+\frac{y^{2 j m}}{j m}, \quad j, n, m \in \mathbb{N}, \quad \text { НОД }(m, n)=1 .
$$

Рассмотрим замены переменных

$$
\begin{aligned}
& x=\alpha r^{m}|\cos \varphi|^{k} \operatorname{sign}(\cos \varphi), \\
& y=\beta r^{n}|\sin \varphi|^{l} \operatorname{sign}(\sin \varphi),
\end{aligned}
$$

где $\alpha, \beta, k, l$-некоторые положительные константы. Система (1.1) принимает вид

$$
r^{\prime}=\frac{r\left(x k q(x, y) \sin ^{2} \varphi+y l p(x, y) \cos ^{2} \varphi\right)}{\sin \varphi \cos \varphi(x m q(x, y)-y n p(x, y))} .
$$

Замены переменных (3.2) действуют в четырех криволинейных секторах плоскости (или в четырех квадрантах $(i-1) \pi / 2 \leqslant \varphi \leqslant i \pi / 2, i=1, \ldots, 4)$, а уравнение (3.3) - это четыре ОДУ, определенные в каждом квадранте.

Рассмотрим первый квадрант (для остальных квадрантов вычисления аналогичны). Константы $\alpha, \beta, k, l$ можно выбрать так, что гамильтониан (3.1) не будет зависеть от переменной $\varphi$. Положим $k=1 / j n, l=1 / j m$, тогда для невозмущенной системы ОДУ, соответствуюшей гамильтониану (3.1), замена (3.2) дает уравнение

$$
r^{\prime}=\frac{r \cos \varphi \sin \varphi\left(m \alpha^{2 j n}-n \beta^{2 j m}\right)}{j m n\left(m \alpha^{2 j n} \cos ^{2} \varphi+n \beta^{2 j m} \sin ^{2} \varphi\right)} .
$$

Поэтому при $m \alpha^{2 j n}=n \beta^{2 j m}$ уравнение (3.4) имеет вид $r^{\prime} \equiv 0$. Положим $\alpha=$ $(j n)^{1 / 2 j n}, \beta=(j m)^{1 / 2 j m}$, тогда $r=H^{1 / 2 j n m}$.

Таким образом, невозмушенная траектория системы (1.1) преобразована в окружность, причем полярный радиус определен в каждом квадранте как некоторая 
(одна и та же) степень гамильтониана (3.1). Для возмущенной системы разложение в ряд Тейлора правой части уравнения (3.3) по степеням $r$ начинается со степени $r$, определяемой возмущением в каждом квадранте, т.е. возмушение в системе (1.1) также определяется некоторой степенью гамильтониана (3.1). Таким образом, аналитическое отображение последования склеивается из четырех отображений последования (вообще говоря, конечной гладкости из-за дробных степеней $\sin \varphi$ и $\cos \varphi)$.

Уравнения в вариациях вычисляются независимо в каждом квадранте, а их решения склеиваются (по правилу дифференцирования композиции функций), что позволяет вычислить производные к кривой $\chi=\{r(0), r(2 \pi)\}$, т.е. фокусные величины.

Таким образом, в данном частном случае дан ответ на вопрос о склеивании транзитных траекторий в криволинейных секторах (см. [1; п. 4.7]).

ПРИмеР 3.1. Для системы уравнений

$$
\dot{x}=y, \quad \dot{y}=-x^{3}+a y^{2}+b x^{3} y+c y^{3}
$$

сделаем замену переменных

$$
x=r \sqrt{|\cos \varphi|} \operatorname{sign}(\cos \varphi), \quad y=\frac{r^{2} \sin \varphi}{\sqrt{2}} .
$$

Здесь отображение последования - это композиция двух отображений:

$$
r\left(-\frac{\pi}{2}\right) \rightarrow r\left(\frac{\pi}{2}\right), \quad r\left(\frac{\pi}{2}\right) \rightarrow r\left(\frac{3 \pi}{2}\right),
$$

где $r(\varphi)$ удовлетворяет уравнениям (3.4).

Начальные значения решений уравнений в вариациях $r_{k}(\varphi)$ в правой полуплоскости берутся в точке $\varphi=-\pi / 2$, т.е. $r_{1}(-\pi / 2)=1, r_{k}(-\pi / 2)=0, k=2,3, \ldots$. В левой полуплоскости начальные значения решений - это значения $r_{k}(\pi / 2), k=$ $1,2, \ldots$, функций в правой полуплоскости. В обеих полуплоскостях $r_{1}^{\prime}=0$, т.е. $r_{1} \equiv 1$ в силу замены $(3.6)$.

Вычислив уравнения в вариациях до четвертого порядка [6], получаем (учитывая, что интеграл от периодической функции по периоду не зависит от фазы):

$$
\int_{0}^{2 \pi} r_{4}^{\prime}(\varphi) d \varphi=r_{4}\left(\frac{3 \pi}{2}\right)=-\frac{32}{7} K\left(\frac{1}{\sqrt{2}}\right)(a b+3 c) \approx-8.47577(a b+3 c)
$$

где $K(1 / \sqrt{2})$ - это полный эллиптический интеграл.

Таким образом, асимптотика отображения последования для системы (3.5) имеет вид

$$
r(2 \pi)=r(0)-\frac{32}{7} K\left(\frac{1}{\sqrt{2}}\right)(a b+3 c) \frac{r(0)^{4}}{4 !}+\cdots .
$$

Гипотеза: необходимое условие центра $a b+3 c=0$ является также и достаточным условием центра точки нуль системы (3.5) [6].

Это условие может быть получено также преобразованием системы (3.5) с помощью формальных рядов [11], однако информация об асимптотике отображения последования при этом теряется. 
ПРИмЕР 3.2 (продолжение примера 2.2). Для системы уравнений (2.7) при $a=0$ сделаем замену переменных (3.6). Тогда $r_{1} \equiv 1$ и

$$
r_{2}^{\prime}(\varphi)=-\frac{\sqrt{2} b}{2} \sin ^{2} \varphi \sqrt{|\cos \varphi|}
$$

Вычисляя интеграл по периоду $2 \pi$ от функции $r_{2}^{\prime}(\varphi)$, получаем вторую фокусную величину (2.8):

$$
r_{2}(0, b)=\frac{8 b}{5}\left(K\left(\frac{1}{\sqrt{2}}\right)-\Pi\left(\frac{1}{2}, \frac{1}{\sqrt{2}}\right)\right) \approx-1.355541 b,
$$

где $K$ и П - полные эллиптические интегралы первого и третьего рода.

Заметим, что появление эллиптических интегралов в примерах 3.1 и 3.2 не случайно (см. [5]).

ПРИмеР 3.3 (продолжение примера 2.4). Для системы уравнений (2.11) при $c=3 a / 2$ сделаем замену переменных $(3.2)$ при $\alpha=(1 / 2)^{1 / 6}, \beta=(1 / 3)^{1 / 4}$, $k=1 / 3, l=1 / 2$. Система (2.11) второй категории, поэтому интегральная кривая укороченной системы не может быть преобразована в окружность заменой (3.2). Тем не менее уравнения в вариациях (рассматриваемые на интервале $[-\pi, \pi]$ ) сушественно упрошаются.

Первое уравнение в вариациях имеет вид

$$
r_{1}^{\prime}(\varphi)=-\frac{a \sqrt{6}}{12} \operatorname{sign}(\sin \varphi) r_{1}(\varphi)
$$

Подставим решение этого уравнения во второе уравнение в вариациях и после несложных преобразований получим уравнение для критического значения параметра $a$, при котором в системе (2.11) рождается предельный цикл

$$
0=\int_{0}^{\pi} \frac{|\cos t|^{2 / 3}\left(3 a \cos t \sin t-\sqrt{6} \cos ^{2} t\right) \exp (-a \sqrt{6} t / 12)}{\sqrt{\sin t}} d t
$$

Решение этого уравнения $a=a_{0}$ найдено с не менее чем тридцатью десятичными знаками. Оно совпадает с критическим значением $a_{0}$, полученньм с той же точностью путем интегрирования уравнений в вариациях примера 2.4. Значение параметра $a=-a_{0}$ также является решением уравнения (3.7), что следует из симметрии укороченной системы (2.11).

Гипотеза: условие центра $a=a_{0}$ не является алгебраическим.

4. Случай ломаной Ньютона, состоящей из двух ребер. Для систем, у которых ломаная Ньютона состоит из двух или более ребер, замены переменных в плоскости $\mathbb{R}^{2}$, сводяшие исследование монодромной особой точки в начале координат к исследованию аналитического отображения последования, не известны и, вероятно, не сушествуют. 
Результаты предыдущего пункта показывают, что в случае возмущений гамильтоновых систем (с одним ребром ломаной Ньютона) существуют замены переменных, при которых гамильтониан играет роль полярного радиуса для всех интегральных кривых одновременно. Покажем, что такие замены переменных существуют в случае двух ребер ломаной Ньютона. Рассмотрим систему уравнений:

$$
\dot{x}=x^{2} y+y^{5}+\cdots, \quad \dot{y}=-x y^{2}-x^{5}+\cdots
$$

как возмущение гамильтоновой системы в смысле п. 3, т.е. пусть мономы, обозначенные многоточием, имеют векторные показатели, лежащие правее ломаной Ньютона системы (4.1).

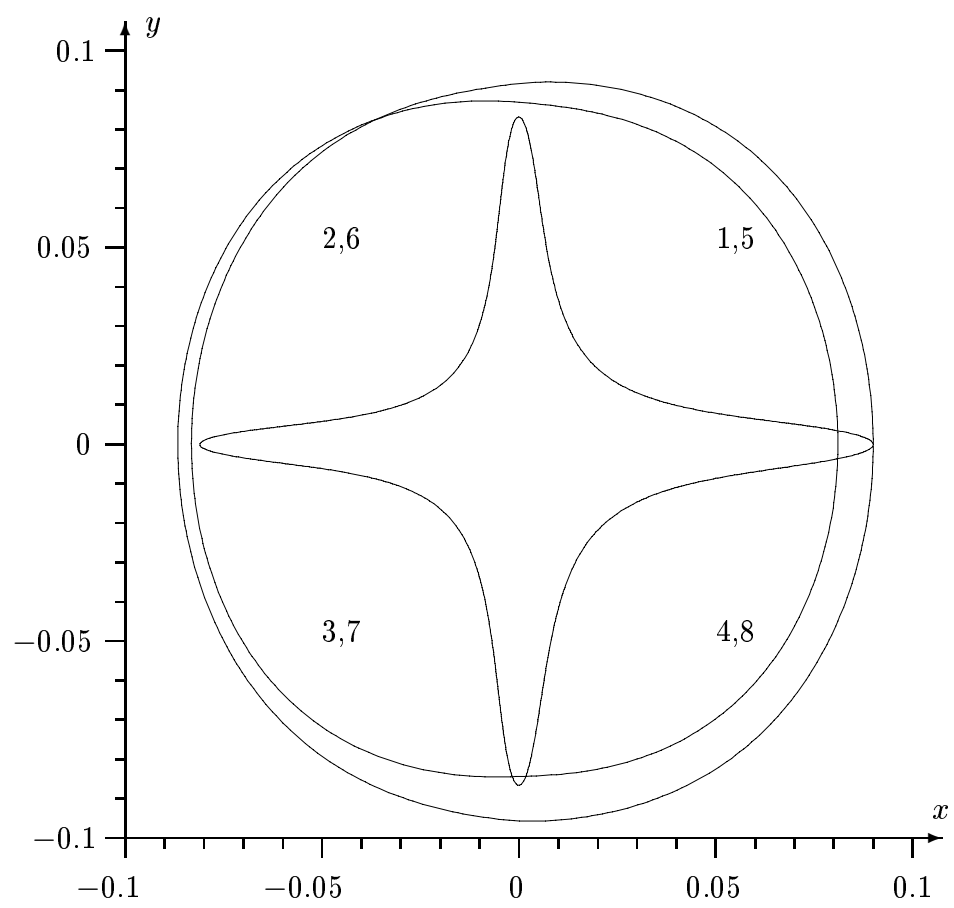

РИс. 2. Интегральная кривая системы (4.1) и ее проекция с поверхности $\mathscr{R}$

Интегральные кривые $x^{6}+3 x^{2} y^{2}+y^{6}=$ const укороченной системы (4.1) образуют крестообразные фигуры и тем больше прилегают к осям координат, чем меньше значение гамильтониана. Поэтому на фазовой плоскости $\{z=x+i y\}$ системы (4.1) будем учитьвать два дополнительных направления, задаваемых прямьми $\{x= \pm y\}$. Отображение $z \rightarrow z^{2}=w=u+i v$ удваивает углы, поэтому интегральные кривые ОДУ, индуцированных системой (4.1) на римановой поверхности $\mathscr{R}=\{z=\sqrt{w}\}$ в проекции на плоскость $\{z\}$, будут выглядеть так же, как у некоторой системы ОДУ с одним ребром ломаной Ньютона на плоскости $\{z\}$ (см. рис. 2). Исходя из этих соображений будем строить замены координат на римановой поверхности $\mathscr{R}$, которую, очевидно, можно реализовать в виде двумерной поверхности в пространстве $\{z, w\} \simeq \mathbb{R}^{4}$. 
Пусть $z=x+i y=r e^{i \phi}$, тогда $z^{2}=w=u+i v=r^{2} e^{2 i \phi}$, т.е. одному обороту вокруг начала координат в плоскости $\{z\}$ соответствует два оборота вокруг особой точки на римановой поверхности $\mathscr{R}$.

Выберем $(u, v)$ в качестве локальных координат на поверхности $\mathscr{R}$. В вешественном виде отображение $z \rightarrow w$ записьвается как $u=x^{2}-y^{2}, v=2 x y$, поэтому отображение проектирования поверхности $\mathscr{R}$ на плоскость $\{z\}$ задается уравнениями

$$
x= \pm \sqrt{\frac{u+\sqrt{u^{2}+v^{2}}}{2}}, \quad y= \pm \sqrt{\frac{-u+\sqrt{u^{2}+v^{2}}}{2}},
$$

где знаки $x$ и $y$ выбираются в зависимости от квадранта плоскости $\{z\}$, в который попадает отображаемая точка.

С точностью до перенормировки $x, y$ и $t$ гамильтониан укороченной системы (4.1) имеет вид

$$
H=3 x^{2} y^{2}+x^{6}+y^{6} \stackrel{\text { def }}{=} r^{6} .
$$

Пусть $x>0, y>0, u>0, v>0$, т.е. рассмотрим первый из восьми секторов поверхности $\mathscr{R}($ так как $0 \leqslant \varphi \leqslant 4 \pi$, см. рис. 2 ). Сделаем замену переменных

$$
u=\sum_{m=1}^{\infty} r^{m} c_{m}(\varphi), \quad v=\sum_{n=1}^{\infty} r^{n} s_{n}(\varphi)
$$

где $r(\varphi), c_{m}(\varphi)$ и $s_{n}(\varphi)$ - аналитические функции в секторе $\varphi \in(0, \pi / 2),|\operatorname{Im}(\varphi)|<$ $\varepsilon$ при малых $|r|$ и $\varepsilon$. Подставим выражения (4.4) в формулы (4.2) и (4.3) и разложим гамильтониан $H$ в ряд Тейлора по переменной $r$. При этом все коэффициенты при членах этого ряда, кроме коэффициента при $r^{6}$, равны нулю. Это дает уравнения, которые позволяют последовательно определить коэффициенты в разложениях (4.4) (не единственным образом).

Таким образом, получаем, что замена переменных

$$
\begin{aligned}
& u=r^{2} \cos ^{2 / 3} \varphi-\frac{4}{3} \frac{r^{4} \sin ^{2} \varphi}{\cos ^{2 / 3} \varphi}+\cdots, \\
& v=\frac{2}{\sqrt{3}} r^{3} \sin \varphi+r^{5} \sqrt{3} \cos ^{2 / 3} \varphi \sin \varphi+\cdots
\end{aligned}
$$

обладает требуемыми свойствами, т.е. подстановка выражений (4.5) в формулы (4.2) обращает формулу (4.3) в тождество.

Аналогично сделав замены переменных в каждом секторе поверхности $\mathscr{R}$, получим, вообще говоря, восемь уравнений $r^{\prime}=f_{j}(r, \varphi), j=1, \ldots, 8$, из решений которых склеивается отображение последования $r(0) \rightarrow r(4 \pi)$. На самом деле, замена (4.5) и аналогичные ей, действующие в других секторах, выбирались таким образом, что отображение последования можно склеить из четырех отображений, действующих в секторах $(8,1),(2,3),(4,5)$ и $(6,7)$ (см. рис. 2$)$.

Пусть в некотором секторе разложения для $u$ и $v$ в замене (4.4) начинаются со степени $r$, равной $m_{0}$ и $n_{0}$ соответственно (т.е. $m_{0}=2$ и $n_{0}=3$ в данном случае). Тогда коэффициенты при $r$ в степени $m_{0}+j-1$ для $u$ и в степени $n_{0}+j-1$ для $v$ участвуют в вычислении уравнений в вариациях начиная с $j$-го порядка, $j=1, \ldots$. 


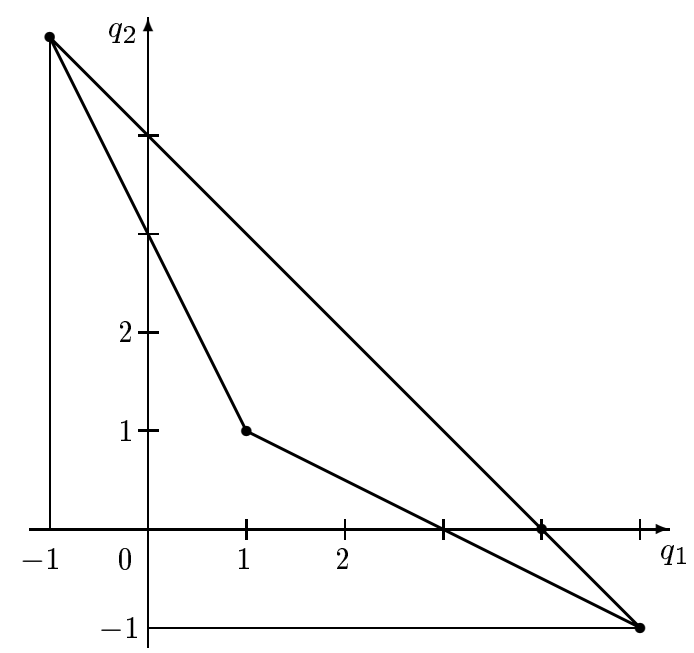

Рис. 3. Многоугольник Ньютона [1] системы (4.6)

Это свойство проверяется прямым вычислением (см. также теорему 2.2). Поэтому в разложениях (4.5) можно оставить только те члены, которые позволяют учесть возмушение в системе (4.1).

ПримеР 4.1. Рассмотрим возмушенную систему

$$
\dot{x}=x^{2} y+y^{5}+a x^{5}, \quad \dot{y}=-x y^{2}-x^{5}+b x^{4} y,
$$

где возмушение выбрано таким образом (см. рис. 3), что условия центра не сводятся к проверке обратимости системы. Кроме того, поскольку возмущению соответствует ближайшая точка к одному из ребер ломаной Ньютона, в замене переменных (4.5) можно оставить только первые члены в разложениях, т.е. использовать замену переменных (3.2) и, соответственно, уравнения (3.3), которые вычисляются в секторах $(8,1),(2,3),(4,5)$ и $(6,7)$ (см. рис. 2$)$.

Во всех секторах согласно замене $(4.5) r_{1}(\varphi) \equiv 1$. В секторах $(2,3)$ и $(6,7)$ получаем $r_{2}(\varphi) \equiv 0$.

Интегральные кривые системы (4.6) на поверхности $\mathscr{R}$ проектируются в кривую на плоскости $\{x, y\}$, проходимую дважды в силу симметрии $(x, y) \rightarrow(-x,-y)$ системы $(4.6)$, поэтому вычисления в секторах $(8,1)$ и $(4,5)$ дают одно и то же.

В секторах $(8,1)$ получаем

$$
r_{2}^{\prime}=-\frac{2}{9} \sqrt{3} \cos ^{1 / 3} \varphi\left(3 a \cos ^{2} \varphi+(a+b) \sin ^{2} \varphi\right) .
$$

Таким образом, асимптотика отображения последования системы (4.6) при $r(0) \rightarrow 0$ имеет вид

$$
r(4 \pi)=r(0)+2 \int_{-\pi / 2}^{\pi / 2} r_{2}^{\prime}(\varphi) d \varphi \frac{r(0)^{2}}{2 !}+\cdots
$$


Вычисляя интеграл в формуле (4.7), получаем

$$
4 \int_{0}^{\pi / 2} r_{2}^{\prime}(\varphi) d \varphi=-6 \frac{2^{1 / 3} \Gamma(2 / 3)^{3}}{7 \pi}(5 a+b) \approx-0.85352480(5 a+b) .
$$

Необходимое условие центра $5 a+b=0$ для системы (4.6) является и достаточным, так как при этом условии возмущенная система также является гамильтоновой.

Результаты этого пункта переносятся практически без изменений на системы ОДУ, порожденные гамильтонианами $H=x^{2} y^{2}+x^{2 n}+y^{2 n}, n=3,4, \ldots$.

Для гамильтонианов с несимметричными ребрами ломаной Ньютона могут потребоваться кусочно гладкие отображения плоскости $\{x, y\}$ на поверхности более обшего вида, которые по аналогии будем называть римановыми.

ПРИмЕР 4.2. Рассмотрим гамильтониан

$$
H=x^{2} y^{2}+x^{6}+y^{8}=r^{6}
$$

и покажем, что существует риманова поверхность, на которой линии уровня гамильтониана (4.8), т.е. интегральные кривые невозмушенной системы ОДУ, порожденной гамильтонианом (4.8), проектируются в концентрические окружности на плоскости $\{x, y\}$.

В пространстве $\mathbb{R}^{4}=\{x, y, u, v\}$ определим кусочно гладкую риманову поверхность $\mathscr{R}_{2}$ двумя уравнениями

$$
u=|x|^{3}-y^{4}, \quad v=x y .
$$

Рассмотрим первый сектор поверхности $\mathscr{R}_{2}$, т.е. $x>0, y>0, u>0, v>0$. Тогда отображение поверхности $\mathscr{R}_{2}$ на плоскость $\{x, y\}$ задается уравнениями

$$
\begin{aligned}
& x=u^{1 / 3}+\frac{1}{3} u^{-2} v^{4}-\frac{5}{9} u^{-13 / 3} v^{8}+\cdots, \\
& y=u^{-1 / 3} v-\frac{1}{3} u^{-8 / 3} v^{5}+\frac{2}{3} u^{-5} v^{9}+\cdots .
\end{aligned}
$$

Введем координаты

$$
u=r^{3} \cos \varphi+\cdots, \quad v=r^{3} \sin \varphi+\cdots .
$$

Тогда $u^{2}+v^{2}=r^{6}-2 x^{3} y^{4}+\cdots=H+o\left(r^{9}\right)$. Разлагая $H=u^{2}+v^{2}+2 x^{3} y^{4}$ в ряд Тейлора по $r$, определяем последующие члены в разложениях (4.9):

$$
\begin{aligned}
& u=r^{3} \cos \varphi+r^{8} \frac{\sin ^{4} \varphi}{\cos ^{4 / 3} \varphi}-\frac{1}{2} r^{13} \frac{\sin ^{8} \varphi}{\cos ^{11 / 3} \varphi}+\cdots, \\
& v=r^{3} \sin \varphi+o\left(r^{13}\right) .
\end{aligned}
$$

В результате получаем $H=r^{6}+O\left(r^{21}\right)$ и т.д.

Автор благодарит А. Д. Брюно за конструктивную критику. 


\section{Список литературы}

1. Брюно А. Д. Локальный метод нелинейного анализа дифференциальных уравнений. М.: Наука, 1979.

2. Медведева Н. Б. Главньй член асимптотики преобразования монодромии // Сиб. матем. журн. 1997. Т. 38. № 1. С. 135-150.

3. Арнольд В.И., Ильяиенко Ю.С. Обыкновенные дифференциальные уравнения // Итоги науки и техники. Т. 1. М.: ВИНИТИ, 1985. С. 7-149.

4. Брюно А. Д. Степенная геометрия в алгебраических и дифференциальных уравнениях. М.: Физматлит, 1998.

5. Ляпунов A. M. Исследование одного из особенных случаев задачи об устойчивости движения // Собр. соч. Т. 2. М.-Л.: ОНТИ, 1956. С. 272-331.

6. Варин В. П. Проблема центра-фокуса и уравнения в вариациях // Препринт, № 9 . Институт прикл. матем. им. М.В. Келдьша. М., 2001.

7. Немыцкий B. В., Степанов В. В. Качественная теория дифференциальных уравнений. М.-Л.: Гостехиздат, 1949.

8. Varin V.P. Degeneracies of periodic solutions to the Beletsky equation // Regul. Chaotic Dyn. 2000. V. 5. № 3. P. 313-328.

9. Ильяиенко Ю.С. Алгебраическая неразрешимость и почти алгебраическая разрешимость проблемы центр-фоокус // Функц. анализ и его прилож. 1972. Т. 6. № 3. С. 30-37.

10. Ван-дер-Варден Б. Л. Современная алгебра, ч. 2. М.-Л.: ОНТИ, 1947.

11. Садовский А. П. О проблеме различения центра и фокуса для систем с ненулевой линейной частью // Дифференц. уравнения. 1976. Т. 12. № 7. С. 1238-1246.

12. Schlomiuk D. Algebraic and geometric aspects of the theory of polynomial vector fields // Bifurcations and periodic orbits of vector fields: Kluwer Acad. Publ. Netherlands, 1993. P. 429-467.

13. Edneral V.F. Computer evaluation of cyclicity in planar cubic system // Proceedings of the ISSAC'97 / ed. W. Küchlin. N.Y.: ACM, 1997. P. 305-309.

14. Варин В. П. Условия центра для систем, близких к гамильтоновьп // Препринт, № 48. Институт прикл. матем. им. М.В. Келдьша. М., 2001.

Институт прикладной математики РАН

им. М.В. Келдьшша
Поступила в редакцию 18.06 .2003 и 15.01 .2004 